\title{
ACTIVIDAD DE FOSFOLIPASAY SUSCEPTIBILIDAD IN VITRO A FLUCONAZOL EN AISLAMIENTOS CLINICOS YAVIARIOS DE Cryptococcus neoformans
}

\author{
(Phospholipase activity and «in vitro» susceptibility to fluconazol \\ in clinical and aviars isolates of Cryptococcus neoformans)
}

\author{
Gabriel Pérez C. (1), Gisela González H. (2), \\ M. Cristina Díaz J. (3) \\ (1). Facultad de Medicina, Universidad de Chile \\ (2). Programa Doctorado en Ciencias Silvoagropecuarias y Veterinarias, Universidad de Chile \\ (3).- Programa de Microbiologia y Micología, ICBM, \\ Facultad de Medicina, Universidad de Chile. \\ mcdiaz@med.uchile.cl
}

Palabras clave: Fosfolipasa, Cryptococcus neoformans, susceptibilidad Fluconazol Key words: Phospholipase, Cryptococcus neoformans, susceptibility Fluconazol

\section{RESUMEN}

Se detectó la actividad de fosfolipasa en 19 cepas clínicas y 17 aviarias de C. neoformans var. neoformans, usando Agar Sabouraud con yema de huevo, incubándose a $37^{\circ} \mathrm{C}$ por 5 dias. Se determinó el índice $P z$ estableciéndose los siguientes rangos: Pz muy alto (0.9-1), alto (0.89-0.80), bajo (0.79-0.70) y muy bajo $(<$ 0.69). El $84 \%$ de las cepas clínicas presentaron índice $\mathrm{Pz}$ muy bajo, el 5\% bajoy 11\% muy alto. Mientras en las cepas aviarias el $82 \%$ presentaron índice muy bajo y un 18\% muy alto. Los valores de Pz promedio fueron muy bajos en todos los aislamientos, sin existir diferencias significativas $(p>0,05)$ entre las cepas clinicas $y$ aviarias, lo que implica una alta actividad enzimática. La susceptibilidad in vitro a Fluconazol se realizó por el método de difusión con discos y el 89,5\% de las cepas clínicas fueron sensibles y el 10,5\% resistentes, mientras en las cepas aviarias, el 59\% fueron sensibles, $29 \%$ sensible dosis dependiente y un $12 \%$ resistentes.

\section{INTRODUCCION}

Cryptococcus neoformans es una levadura capsulada que pertenece a los Basidiomycota (Garau, 2002) y que posee diversos factores de virulencia, entre los cuales se encuentra la fosfolipasa y por ende la produc-

Recibido el 30 Octubre 2008

Aceptado el 5 Diciembre 2008
ABSTRACT

Phospholipase activity was determined to 19 clinical and 17 aviars trains of $\boldsymbol{C}$. neoformans var. neoformans, incubating the yeast for 5 days at $37^{\circ} \mathrm{C}$ on Sabouraud Agar supplemented with egg yolk. Pz values were determined and the following ranges were established:very high (0.9-1), high (0.89-0.80), low (0.79-0.70) and very low $(<0.69)$. The $84 \%$ of the clinical isolates showed Pz values very low ( $5 \%$ ) and $11 \%$ very high. On the other hand, the $82 \%$ of the aviars strains presented $P z$ values very low and $18 \%$ very high. Average Pz values were very low in all isolates, there were no statiscally significant differences $(p>0.05)$ impliying a high enzymatic activity. Susceptibility in vitro testing to Fluconazole was performed by a disk diffusion method (M44-A).The 89.5\% of the clinical isolates were susceptible and $10.5 \%$ resistant, while in avian strains, 59\% were susceptible, 29\% susceptible dose dependent and $12 \%$ resistant.

ción de esta enzima extracelular se relaciona eventualmente con patogenicidad (Ghannoum, 2000).

Paralelamente al aumento de la población en estado de inmunodepresión, la infección por $\boldsymbol{C}$. neoformans ha emergido como una causa importante de morbilidad y mortalidad, afectando especialmente a pacientes con SIDA en etapa avanzada. Actualmente, representa la causa más común de meningitis fúngica en el mundo, y es una de las levaduras más frecuentemente asociadas a infección fún- 
gica invasora (García-Ruiz et al., 2004; Warnock, 2007).

La virulencia de esta levadura es un tema ampliamente estudiado, diversas investigaciones han descrito factores de virulencia para $\boldsymbol{C}$. neoformans, entre los que se encuentran la actividad fosfolipasa extracelular, la producción extracelular de melanina y de prostaglandinas, la producción de cápsula de polisacáridos y la habilidad de crecer a $37^{\circ} \mathrm{C}$ (Vartivarian et al.,1993; Vidotto et al., 1996; Chen, 1997; Casadevall et al., 2000; Cox et al., 2001; Noverr et al., 2001; Perfect, 2006; Bovers et al., 2008).

La fosfolipasa puede dañar las membranas celulares del hospedador generando disfunción y/o disrupción física de las membranas (Ghannoum, 2000) y jugar un rol importante en la patogénesis de este hongo oportunista (Vidotto et al., 1998). Más aún, la posible asociación de producción de fosfolipasa por cepas de Cryptococcus virulentas ya fue reportada por Chen et al. (1998).

Con respecto a la actividad enzimática extracelular de fosfolipasa, Ganendren et al. (2006), observaron que esta es necesaria para la iniciación de la infección pulmonar, debido a que aumenta considerablemente la adhesión de la levadura al epitelio pulmonar. Santangelo et al. (2004), demostraron que la actividad fosfolipasa promueve la sobrevida y replicación de $\boldsymbol{C}$. neoformans en macrófagos, lo que permite la iniciación de la infección pulmonar intersticial y que la fagocitosis mononuclear es el vehículo para la diseminación sistémica de esta levadura. Shea et al. (2006), observaron que la actividad fosfolipasa protege a $\boldsymbol{C}$. neoformans de la acción lítica de las enzimas del fagolisosoma del macrófago activado, y que esto aumenta la diseminación de la levadura al sistema nervioso.

La actividad fosfolipasa extracelular puede detectarse en Candida albicans y $\boldsymbol{C}$. neoformans mediante diversas metodologías, tales como agar malta más yema de huevo, agar Sabouraud glucosado más yema de huevo (SEA) ( Price et al., 1982; Chen et al., 1997; Echeverría et al., 2002) y pruebas específicas colorimétricas y radiométricas (Banno et al., 1985; Ghannoum et al., 2000). El medio de cultivo SEA constituye un método semicuantitativo, de amplia utilización y de mayor sensibilidad para la detección de esta enzima en $\boldsymbol{C}$. neoformans que otros similares (Chen et al., 1997 y Echeverría et al., 2002).

La sensibilidad de $\boldsymbol{C}$. neoformans a antifúngicos utilizados en clínica para su tratamiento puede ser evaluada in vitro mediante técnicas estandarizadas tales como métodos de dilución en caldo, Etest y métodos de difusión en agar, siendo este último el de mayor uso en la actualidad debido a su elevada precisión y exactitud (Barry et al. 2002; Pfaller et al., 2004), y su mejor relación costoefectividad en comparación con otros métodos.

Actualmente, no existe evidencia de que esta infor- mación pueda correlacionarse con la respuesta terapéutica obtenida in vivo; sin embargo, un valor in vitro elevado de CIM puede predecir el fallo terapéutico de un antifúngico (Dannaoui et al., 2006).

El objetivo de este estudio es detectar la actividad fosfolipasa en aislamientos clínicos y aviarios de $\boldsymbol{C}$. neoformans y evaluar su susceptibilidad in vitro a Fluconazol

\section{MATERIALES Y METODOS}

Aislados de $\boldsymbol{C}$. neoformans (Cn). Se utilizaron 19 cepas clínicas de $C n$, aisladas de muestras de sangre y LCR de pacientes con criptococosis, obtenidas en diversos centros hospitalarios del país y 17 cepas de $C n$ obtenidas de deyecciones secas expuestas al medio ambiente de aves exóticas de 3 colecciones privadas y de psitácidas de un centro de rehabilitación de fauna nativa de la Región Metropolitana.

Detección de la actividad fosfolipasa. La detección y cuantificación de la actividad fosfolipasa extracelular se realizó de acuerdo al método de Price (1982), con algunas modificaciones. Las cepas aisladas previamente fueron sembradas en placas de Agar Sabouraud sin antibiótico e incubadas a $37^{\circ} \mathrm{C}$ durante $48 \mathrm{hrs}$, posteriormente se inocularon en placas con medio de cultivo SEA (agar sabouraud glucosado con yema de huevo estéril) e incubadas a $37{ }^{\circ} \mathrm{C}$ durante 5 días. Se midieron los diámetros de las colonias y el diámetro total de la colonia más la zona de hidrólisis producida por la actividad enzimática y se calculó la relación entre ambos valores (índice Pz de Price). A mayor valor de Pz, menor producción de fosfolipasa por la colonia. La lectura se realizó en todas las muestras a los 5 días. Cada aislado de $\boldsymbol{C}$. neoformans se clasificó de acuerdo al valor de su coeficiente $\mathrm{Pz}$ como sigue: Pz muy alto (0.9-1); Pz alto (0.89-0.80); Pz bajo (0.790.70); Pz muy bajo $(<0.69)$.

Susceptibilidad in vitro a Fluconazol : se evaluó mediante el método de difusión con discos de $25 \mu \mathrm{g}$ de Fluconazol (Becton Dickinson), de acuerdo a las recomendaciones del documento M44-A de CLSI, utilizando medio Mueller-Hinton con glucosa al $2 \%$ y azul de metileno (MHGAM). Las 36 cepas aisladas previamente fueron sembradas en placas de Agar Sabouraud sin antibiótico e incubadas a $37^{\circ} \mathrm{C}$ durante $48 \mathrm{hrs}$, el inóculo se preparó en solución salina y se ajustó a 0.5 en la escala Mc Farland por medida espectrofotométrica. Se incluyó Candida krusei ATCC 6258 y Candida parapsilosis ATCC22019 como cepas de control de calidad.

La interpretación de los resultados se llevó a 
Tabla 1: Actividad de fosfolipasa en cepas clínicas y aviarias de $C$. neoformans

\begin{tabular}{|c|c|c|c|c|c|}
\hline \multirow{2}{*}{ Valor Pz } & \multirow{2}{*}{$\begin{array}{c}\text { Actividad } \\
\text { fosfolipasa } \\
\text { (Índice Pz) }^{\text {Ín }}\end{array}$} & \multicolumn{4}{|c|}{ Cepas de $C$ neoformans } \\
\hline & & n & $\begin{array}{r}\text { nicas } \\
(\%)\end{array}$ & $\mathbf{n}^{A}$ & $\begin{array}{l}\text { rias } \\
(\%)\end{array}$ \\
\hline$<0.69$ & ++++ & 16 & 84,21 & 14 & 82,35 \\
\hline $0.70-0.79$ & +++ & 1 & 5,26 & - & - \\
\hline $0.80-0.89$ & ++ & - & & - & - \\
\hline $0.90-1.0$ & + & 2 & 10,53 & 3 & 17,65 \\
\hline
\end{tabular}

cabo de acuerdo a la tabla $\mathrm{N}^{\mathrm{o}} 1$ de documento M44-A.

Según el diámetro del halo de inhibición del crecimiento de la colonia; cada aislado frente al disco de $25 \mu \mathrm{g}$ de Fluconazol se clasificó como sensible $(\geq 19 \mathrm{~mm})$, sensible dosis dependiente $(15-18 \mathrm{~mm})$, o resistente $(\leq 14 \mathrm{~mm})$.

Análisis Estadístico. Con el objeto de comparar el valor Pz del día 5 de incubación de las cepas de $C n$ de origen humano y aviar, se utilizó la prueba estadística de Mann-Whitney con cálculo de U para dos muestras independientes.

\section{RESULTADOS}

Detección actividad fosfolipasa. En las 19 cepas clínicas analizadas, los valores $\mathrm{Pz}$ al día 5 de incubación variaron en un rango de 0,26 a 1 (Promedio $\mathrm{Pz}=0,44 \pm 0,21$ y coeficiente de variación (CV) de 48\%). Cerca del $90 \%$ presentó un índice $\mathrm{Pz}$ bajo o muy bajo (1 y 16 respectivamente) y 2 muestras presentaron índice Pz muy alto $(0.915$ y 1$)$. Sólo en una de las cepas, no se detectó actividad fosfolipasa $(\mathrm{Pz}=1)$

En las 17 cepas aisladas de deyecciones de aves, los valores $\mathrm{Pz}$ al día 5, variaron entre 0,28 y 1 (promedio $\mathrm{Pz}=0,49 \pm 0,264$ y CV de 54\%). Catorce cepas presentaron un Pz muy bajo (82\%) y 3, muy alto (18\%). En estas tres cepas no se detectó actividad fosfolipasa.

No hubo diferencias significativas entre los aislados clínicos y los aviarios mediante la prueba de Mann Whitney $(\mathrm{p}>0,05), \mathrm{U}=144$.

Susceptibilidad in vitro a Fluconazol . En 19 cepas clínicas, $17(89,47 \%)$ fueron sensibles mientras que 2 (10,53\%) resultaron resistentes. En 17 aislamientos aviarios, 10 fueron sensibles $(58,8 \%), 5(29,4 \%)$ sensibles dosis dependiente y $2(11,8 \%)$ resistentes (Tabla 2$)$.

\section{DISCUSION}

La actividad fosfolipasa extracelular ha sido implicada en la patogénesis de infecciones bacterianas y fúngicas Ghannoum et al.(2000), existen numerosas publicaciones en que se ha detectado esta actividad en levaduras, principalmente en Candida albicans $\boldsymbol{y} \boldsymbol{C}$. neoformans usando diferentes métodos para medirla (Price et al.,1982; Chen et al., 1997; Echeverría et al., 2002). Entre éstos, el método desarrollado por Price resulta conveniente y sencillo de utilizar en un gran número de aislados (Vidotto et al., 1998). Además la correlación entre el valor Pz y la actividad fosfolipasa ya fue demostrada (Price et al., 1982).

En el presente estudio, no hubo diferencias significativas en los valores $\mathrm{Pz}$ entre las cepas de ambos grupos, lo que concuerda con lo comunicado por Chen et al. (1997), y difiere de lo reportado por Vidotto et al. (1998), quienes si encontraron diferencias significativas.

En nuestro estudio, las cepas aviarias presentaron, con respecto a los aislados clínicos un $\mathrm{Pz}$ promedio mayor $(0,49$ vs 0,44$)$; y un mayor número de aislados sin detección de fosfolipasa (15,8\% vs 5,3\%). Esta tendencia se mantuvo igual al estudio de Vidotto et al. (1998), donde las cepas

Tabla 2: Susceptibilidad in vitro a Fluconazol en aislamientos clínicos y ambientales de $C$. neoformans

\begin{tabular}{|c|c|c|c|c|}
\hline \multirow{2}{*}{$\begin{array}{c}\text { Cepas de } \\
\text { C. neoformans }\end{array}$} & \multirow[t]{2}{*}{$\mathbf{N}$} & \multicolumn{3}{|c|}{$\begin{array}{c}\text { Susceptibilidad in vitro a } \\
\text { Fluconazol }^{\mathrm{a}}\end{array}$} \\
\hline & & $\% \mathrm{~S}$ & $\%$ SDD & $\% R$ \\
\hline Clínicas & 19 & 89,47 & - & 10,53 \\
\hline Aviarias & 17 & 58,82 & 29,41 & 11,77 \\
\hline $\begin{array}{l}\text { Sensible (S, } \\
(\text { SDD,15-18mm), }\end{array}$ & & sen & $\begin{array}{l}\text { dosis } \\
\mathrm{mm})\end{array}$ & \\
\hline
\end{tabular}


aviarias también presentaron, con respecto a las cepas clínicas, un valor $\mathrm{Pz}$ promedio mayor $(0,941$ y 0,652 respectivamente) y un mayor número de cepas sin detección de fosfolipasa ( $74 \%$ y 4,76\%, respectivamente).

La mayoría de las cepas estudiadas fueron sensibles a Fluconazol (89,5\% clínicas, 59\% aviarias), pero en las cepas aviarias se detectó un mayor número de cepas sensibles dosis dependiente (29\%). Estos resultados son similares a los obtenidos por Pfaller et al. (2007), en cepas clínicas, que reportan una sensibilidad de $78 \%$ a Fluconazol. Referente a las cepas aviares, un estudio identificó los aislados obtenidos a partir de un brote de Cryptococosis en psitácidas en Brasil como resistentes a Fluconazol (Raso et al., 2004), mientras que en Malasia, todos los C. neoformans aislados de deyecciones aviares fueron sensibles al antifúngico (Tay et al., 2005).

En base a lo expuesto podemos concluir que los valores de Pz promedio fueron muy bajos en aislamientos clínicos y aviarios de $\mathrm{C} n$, lo que se traduce en un nivel de actividad fosfolipasa alta y sugiere un rol de la enzima en la invasión al hospedero. En muy pocas cepas no se detectó la producción de fosfolipasa.

\section{REFERENCIAS}

Banno, Y.; Yamada, T. \& Nozawa, Y. (1985). Secreted phospholipases of the dimorphic fungus, Candida albicans; separation of the three enzymes and some biological properties. Sabouraudia 23:47-54

Barry, A.; Pfaller, M.; Rennie, R.; Fuchs, P.; Brown, S. (2002). Precision and Accuracy of Fluconazole Susceptibility Testing by Broth Microdilution, Etest, and Disk Diffusion Methods. Antimicrobial Agents and Chemotherapy 46:1781-1784

Bovers, M.; Hagen, F.; Boekhout, T. (2008). Diversity of the Cryptococcus neoformans- Cryptococcus gattii species complex. Rev. Iberoam. Micol. 25:S4-S12

Casadevall, A.; Rosas, A.; Nosanchuk, J. (2000). Melanin and virulence in Cryptococcus neoformans. Current Opinion in Microbiology 3:354-358

Cox, G.; McDade, H.; Chen, S.; Tucker, S.; Gottfredsson, M.; Wright, L.; Sorrell, T.; Leidich, S.; Casadevall, A.; Ghannoum, A.; Perfect, J. (2001). Extracellular phospholipase activity is a virulence factor for Cryptococcus neoformans. Molecular Microbiology 39:166-175

Chen, S.; Muller, M.; Zhou, Z.; Wright, L.; Sorrell, T. (1997) Phospholipase activity in Cryptococcus neoformans: a new virulence factor?. J. Infect. Dis. 175:414-420

Dannaoui, E.; Abdul, M.; Arpin, M.; Michel-Nyugen, A.; Piens, A.; Favel, A.(2006 ). Results obtained with various antifungal susceptibility testing methods do not predict early clinical outcome in patients with cryptococcosis Antimicrobial Agents and Chemotherapy 50:2464-2470

Echeverría, A.; Durante, A.; Arechavala, A.; Negroni, R.
(2002). Estudio comparativo de dos medios de cultivo para la detección de la actividad fosfolipasa en cepas de Candida albicans y Cryptococcus neoformans. Rev. Iberoam. Micol. 19:95-98

Ganendren, R.; Carter, E.; Sorrell, T.; Widmer, F.; Wright, L. (2006). Phospholipase B activity enhances adhesion of Cryptococcus neoformans to a human lung epithelial cell line. Microbes and Infection 8:1006-1015

Garau, M. \& Del Palacio, A. (2002). Artritis por Cryptococcus neoformans en receptor de transplante renal. Rev. Iberoam. Micol. 19:186-189

Ghannoum, M. (2000). Potential role of phospholipases in virulence and fungal pathogenesis. Clin. Microbiol. Rev. Jan: 122143

García-Ruiz, J.C.; Amutio, E. \& Pontón, J. (2004). Infección fúngica invasora en pacientes inmunodeficientes. Rev. Iberoam. Micol. 21:55-62

Noverr, M.; Phare, S.; Toews, G.; Coffey, M.; Huffnagle, G.(2001). Pathogenic Yeasts Cryptococcus neoformans and Candida albicans produce Immunomodulatory Prostaglandins. Infection and Immunity May:2957-2963

Perfect, J.R. (2006). Cryptococcus neoformans: the yeast that likes it hot. FEMS Yeast Res 6:463-468

Pfaller, M.; Hazen, K.; Messer, S.; Boyken, L.; Tendolkar, S.; Hollis, R.; Diekemal, D. (2004). Comparison of Results of Fluconazole Disk Diffusion Testing for Candida Species with Results from a Central Reference Laboratory in the ARTEMIS Global Antifungal Surveillance Program. Journal of Clinical Microbiology 8:3607-3612

Pfaller. M.; Diekema, D.; Gibbs, D.; Newell, V.; Meis, J.; Gould, I.; Fu, W.; Colombo, A.; Rodriguez-Noriega, E.; the Global Antifungal Surveillance Group. (2007). Results from the ARTEMIS DISK Global Antifungal Surveillance Study, 1997 to 2005: an 8.5-Year Analysis of Susceptibilities of Candida Species and Other Yeast Species to Fluconazole and Voriconazole Determined by CLSI Standardized Disk Diffusion Testing. Journal of Clinical Microbiology 45,6:1735-1745

Price, M.; Wilkinson, I. \& Gentry, L. (1982). Plate method for detection of phospholipase activity in Candida albicans. Sabouraudia 20:7-14

Raso, T.F.; Werther, K.; Miranda, E.T.; Mendes-Giannini, M.J. (2004). Cryptococcosis outbreak in psittacine birds in Brazil. Med. Mycol. 42:355-362

Santangelo, R., Zoellner, H.; Sorrell, T.; Wilson, C.; Donald, C.; Djordjevic, J.; Shounan, Y.; Wright, L. (2004). Role of extracellular phospholipases and mononuclear phagocytes in dissemination of Cryptococcosis in a murine model. Infection and Immunity Apr:2229-2239

Shea, J.; Kechichian, T.; Luberto, C.; Del Poeta, M. (2006) The Cryptococcal Enzyme Inositol PhosphosphingolipidPhospholipase C Confers Resistance to the Antifungal Effects of Macrophages and Promotes Fungal Dissemination to the Central Nervous System. Infection and Immunity; Oct: 5977-5988

Tay, S.T.; Chai, H.C.; Na, S.L.; Hamimal, H.; Rohani, M.Y. 
M.; Soo-Hoo,T.S. (2005). The isolation, characterization and antifungal susceptibilities of Cryptococcus neoformans from birds excreta in Klan Valley, Malaysia. Mycopathologia 159:509-513

Vartivarian, S.E.; Anaissie, E.J.; Cowart, R.E.; Sprigg, H.A.; Tingler, M.J.; Jacobson, E.S. (1993). Regulation of cryptococcal capsular polysaccharide by iron. J. Infect. Dis. 161:186-190

Vidotto, V.; Sinicco, A.; Di Fraia, D.; Cardaropoli, S.; Aoki, S.; Ito-Kuwa, S. ( 1996) Phospholipase activity in Cryptococcus neoformans. Mycopathologia 136:119-123
Vidotto, V.; Leone, R.; Sinicco, A.; Ito-Kuwa, S.; Criseo, G. (1998). Comparison of phospholipase production in Cryptococcus neoformans isolates from AIDS patients and bird droppings. Mycopathologia 142:71-76

Warnock, D. (2007). Trends in the epidemiology of invasive fungal infections. Jpn. J. Med. Mycol.; 48:1-12 